\section{Scleral fixation of Ahmed glaucoma valve tube tip for adjustment of cornea-touching malposition}

KT Ma', JH Kim², GJ Seong ${ }^{3}$, DS Jang ${ }^{4}$ and $\mathrm{CY} \mathrm{\textrm {Kim } ^ { 3 }}$

\begin{abstract}
Purpose Tube-corneal touch occurring after Ahmed glaucoma valve (AGV) implantation is conventionally treated by tube cutting or tube transposition from the original pathway. However, in some cases, tube cutting is insufficient, and rearranging the pathway of the tube through a new sclera tunnel, ciliary sulcus, or pars plana is not feasible, as the conjunctiva and sclera covering the tube are difficult to be redissected. So, we propose a novel technique that repositions malpositioned AGV tube using scleral fixation and its successful applications in two patients with tube-corneal touch.

Methods (A) A scleral flap is made at the point for scleral fixation. (B) The anterior chamber is maintained using an anterior chamber maintainer. The incision is made immediately above the tube entering the anterior chamber and the tube end is flipped out using a Sinskey. (C) A double-armed 10/0 prolene straight needle is penetrated through the tube end. The leading needle enters the anterior chamber through the previously made incision and is pulled through the scleral flap. (D) The tube tip and the second needle of the double-armed 10/0 prolene straight needle also enter the anterior chamber through the previously made incision and the second needle is pulled through the scleral flap. The tube end is extended to be parallel to the cornea surface. Results Patients maintained good tube positioning without any serious complications during average of $\mathbf{1 5}$ months of follow-up after operation.

Conclusion We believe that our method is a simple and minimally invasive surgical
\end{abstract}

method for treating AGV tube touching of the corneal endothelium. However, considering the limited number of cases studied and the short follow-up period, a larger group with a longer follow-up period is necessary.

Eye (2014) 28, 23-25; doi:10.1038/eye.2013.214; published online 4 October 2013

Keywords: Amhed glaucoma valve; corneal touch; scleral fixation

\section{Introduction}

Recently, the usage of highly effective intraocular pressure (IOP) lowering eye drops has markedly reduced the incidence of glaucoma surgery. ${ }^{1,2}$ However, there are some cases in which IOP control through the use of eye drop is not sufficient, and surgery is needed. Ahmed glaucoma valve (AGV) implantation is one available option for glaucoma surgery; however, tube touching of the corneal endothelium can occur in some cases. ${ }^{1}$ In these cases, removal and reinsertion of the tube through a new pathway is usually the treatment of choice, ${ }^{2}$ but the fragility of the patients' eyes makes additional surgeries challenging. We propose a novel technique of repositioning the tube with sclera fixation, which does not require dissection or retrieval of the tube from the original sclera pathway. We applied this technique in two patients with tube-corneal touch, and successfully corrected the condition.

\section{Materials and methods}

This operation is performed under sub-Tenon's anesthesia on the inferior nasal side.
${ }^{1}$ Jeil Eye Clinic, Gyunggi-do, Korea

${ }^{2}$ Siloam Eye Hospital, Seoul, Korea

${ }^{3}$ Department of Ophthalmology, Institute of Vision Research, Yonsei University College of Medicine, Seoul, Korea

${ }^{4}$ Department of Sculpture, Hongik University, Seoul, Korea

Correspondence: CY Kim, Department of Ophthalmology, Institute of Vision Research, Yonsei University College of Medicine, Seoul 120-752, Korea.

Tel: +82 222283570

Fax: +82 23120541

E-mail: kcyeye@yuhs.ac

Received: 25 June 2013 Accepted in revised form: 23 August 2013 Published online: 4 October 2013 

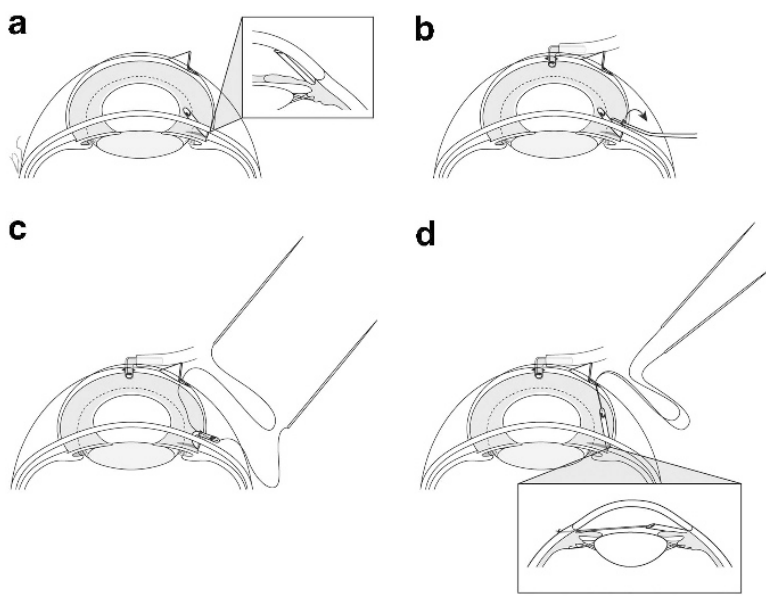

Figure 1 (a) A scleral flap is made at the point for scleral fixation. (b) The anterior chamber is maintained using an anterior chamber maintainer. The incision is made immediately above the tube entering the anterior chamber and the tube end is flipped out using a Sinskey. (c) A double-armed 10/0 prolene straight needle is penetrated through the tube end. The leading needle enters the anterior chamber through the previously made incision and is pulled through the scleral flap. (d) The tube tip and the second needle of the double-armed 10/0 prolene straight needle also enter the anterior chamber through the previously made incision and the second needle is pulled through the scleral flap. The tube end is extended to be parallel to the cornea surface.

A conjunctival incision is made at the site of scleral fixation of the tube (Figure 1a). A triangular-shaped half-thickness scleral flap is made at the point of incision with a \#22 beaver blade. Although the anterior chamber is maintained with an anterior chamber maintainer (Alcon Grieshaber, Schaffhausen, Switzerland) and a BSS instillation is made through the maintainer, a corneal step wound is made immediately above the tube entering the anterior chamber using a diamond knife (Figure 1b). The end of the tube is pulled out through the puncture site using a Sinskey, and a double-armed 10/0 prolene straight needle (Ethicon Inc., Somerville, NJ, USA) is penetrated through the tube end. The leading needle enters the anterior chamber through the previously made incision and is pulled through the scleral flap (Figure 1c). The tube tip and the second needle of the double-armed 10/0 prolene straight needle also enter the anterior chamber through the previously made incision and the second needle is pulled through the scleral flap. The tube end is extended to be parallel to the cornea surface (Figure 1d). After scleral fixation of the tube, the partial thickness scleral flap is closed with a 10-0 nylon suture. The incised conjunctiva and Tenon's capsule are closed with 8-0 vicryl interrupted sutures. Antibiotics and steroid eye drops are used four times a day for 3 weeks after the operation.

\section{Results}

Case 1

A 52-year-old female patient was diagnosed with chronic angle closure glaucoma. After receiving cataract surgery in 2006, the anterior chamber of her right eye became very shallow, and she received AGV (FP 7) implantation due to uncontrolled IOP. She maintained an IOP under $16 \mathrm{~mm} \mathrm{Hg}$ with Goldmann applanation tonometer after the AGV implantation, but corneal touch of the AGV tube end and localized corneal edema developed 4 years after the last operation (Figure 2). Her corrected distance visual acuity (CDVA) was 0.02 and the conjunctiva was very thin, so redissection of the conjunctiva for tube transposition did not seem appropriate.

AGV tube repositioning with sclera fixation was performed as previously described (Figure 3). After the procedure, her CDVA was 0.1 and IOP was $12 \mathrm{~mm} \mathrm{Hg}$ (with brimonidine and timolol-dorzolamide fixed combination). The corneal edema disappeared, and the patient maintained good tube positioning without any serious complications during 18 months of follow-up after the last operation.

\section{Case 2}

A 50-year-old female who had received glaucoma surgery when she was 6 years old was referred to Severance Hospital for cataract surgery. There was no medical record of the specific surgery she had received. Her CDVA was FC $20 \mathrm{~cm}$ and IOP was $13 \mathrm{~mm} \mathrm{Hg}$. Sector iridectomy was noted on the superior side. She received cataract surgery combined with iridoplasty in 2010. One month after cataract surgery, IOP reached $30 \mathrm{~mm} \mathrm{Hg}$ and was uncontrolled even with glaucoma medications, so AGV implantation was performed. After the operation, IOP was $13 \mathrm{~mm} \mathrm{Hg}$, but tube touching of endothelium was noted one month after surgery. As the size of the eyeball and thinning and weakness of the sclera was the main cause of the tube touching, the patient received AGV tube repositioning as previously described. Good IOP maintenance (8-12 $\mathrm{mm} \mathrm{Hg}$ with latanoprost and timolol-dorzolamide fixed combination) was achieved without serious complications over 12 months of follow-up.

\section{Discussion}

Even after successful implantation of a glaucoma drainage device, many complications may occur over the course of follow-up. Tube-corneal touch or corneal decompensation is one of the complications after AGV implantation. ${ }^{1,3-6}$ Patients who have a shallow anterior chamber, corneal guttata, corneal edema, or who have 


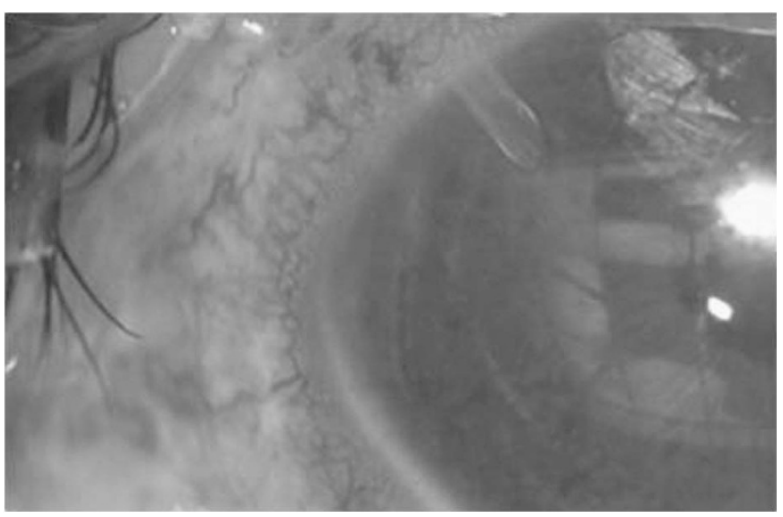

Figure 2 Ahmed glaucoma tube end touching the corneal endothelium and causing localized edema.

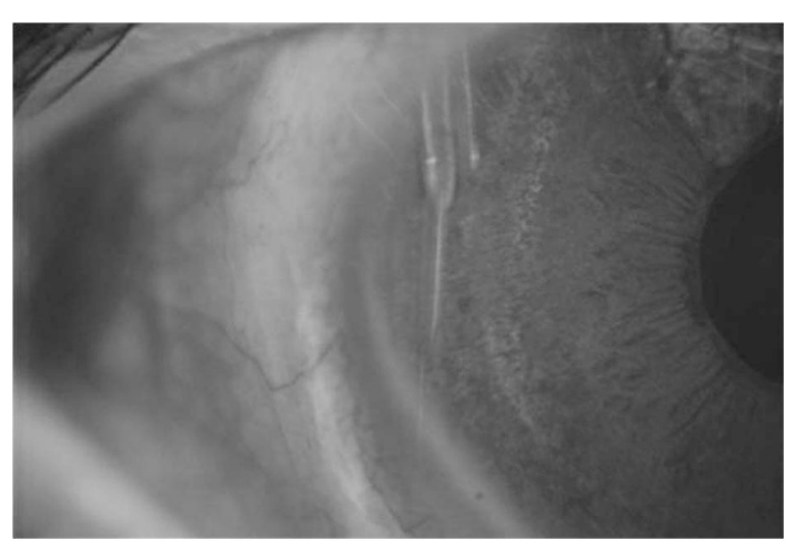

Figure 3 Ahmed glaucoma tube end extended to be parallel to the corneal endothelium by sclera fixation.

previously received corneal transplant may be more prone to corneal touch by the AGV tip. ${ }^{2}$

Many different approaches to address the problem of corneal touch have been reported. Conventional treatment of corneal touch after AGV implantation through the anterior chamber involves cutting the long tube or repositioning the tube through a new sclera pathway, ciliary sulcus, ${ }^{2}$ or pars plana. However, cutting the tube end may not be the best method for avoiding corneal touch in cases of severe tube-corneal touch. In addition, reopening the conjunctiva and sclera for repositioning of the tube through other routes might not be possible because of a very thin or scarred conjunctiva. Dissection of the conjunctiva or sclera might also affect the function of the implanted drainage device. In such cases, repositioning the tube without reopening the conjunctiva or sclera near the drainage device would be ideal. In this paper, we introduce a novel and simple method of repositioning the tube with sclera fixation that does not require dissecting or retrieving the tube from the original sclera pathway. This method is easy to learn and the surgical procedures are less invasive to the globe of the eye than previous reported methods. ${ }^{1,2,6}$ As shown in Figure 1, this method involves making an incision right above the tube entering the anterior chamber and 'flipping' out the tube end using a Sinskey. The rest of the procedure for sclera fixation is the same as in previously described surgical techniques. ${ }^{7}$

In conclusion, we believe that our method is a simple and minimally invasive surgical method for treating AGV tube touching of the corneal endothelium. However, considering the limited number of cases studied and the short follow-up period, a larger group with a longer follow-up period is necessary.

\section{Summary}

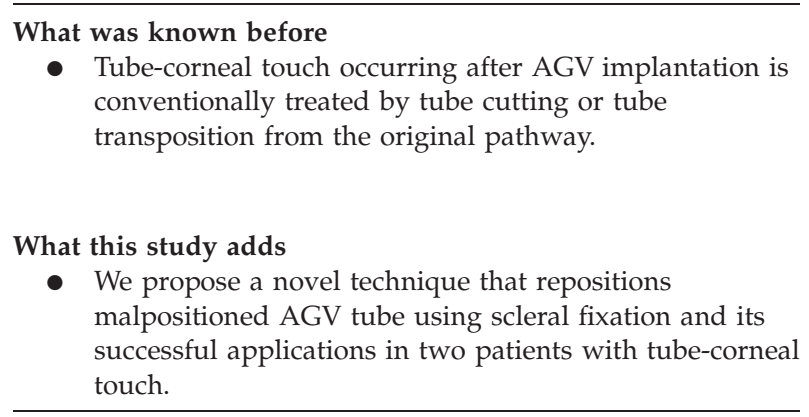

\section{Conflict of interest}

The authors declare no conflict of interest.

\section{References}

1 Sarkisian SR. Tube shunt complications and their prevention. Curr Opin Ophthalmol 2009; 20: 126-130.

2 Weiner A, Cohn AD, Balasubramaniam M, Weiner AJ. Glaucoma tube shunt implantation through the ciliary sulcus in pseudophakic eyes with high risk of corneal decompasation. J Glaucoma 2010; 19: 405-411.

3 Law SK, Coleman AL, Caprioli J. Dynamic tube movement of Amhed glaucoma valve. J Glaucoma 2009; 18: 628-631.

4 Smith MF, Doyle JW, Ticrney JW. A comparison of glaucoma drainage implant tube coverage. J Glaucoma 2002; 11: 143-147.

5 Bersudsky V, Trevino A, Rumelt S. Management of endothelial decompensation because of glaucoma shunt tube touch by descemet membrane endothelial keratoplasty and tube revision. Cornea 2011; 30: 709-711.

6 Sarkisian SR, Netland PA. Tube extender for revision of glaucoma drainage implants. J Glaucoma 2007; 16: 637-639.

7 Ma KT, Kim JH, Kim NR et al. Scleral fixation of standard capsular tension ring and in-the-bag intraocular lens implantation in patients with severe lens subluxation. Ophthalmic Surg Lasers Imaging 2012; 43(6): 504-507. 